\title{
Clinical Characteristics of Temporal Bone Metastases
}

\author{
Kunho Song ${ }^{1}$ Ki-Wan Park ${ }^{1} \cdot$ Jae-Hyung Heo ${ }^{1} \cdot$ Ik-Chan Song $^{2} \cdot$ Yong-Ho Park $^{1} \cdot$ Jin Woong Choi ${ }^{1}$ \\ Departments of ${ }^{1}$ Otorhinolaryngology-Head and Neck Surgery and ${ }^{2}$ Internal Medicine, \\ Chungnam National University Hospital, Chungnam National University College of Medicine, Daejeon, Korea
}

Objectives. The purposes of this study were to evaluate the clinical characteristics of temporal bone metastasis (TBM) and to determine whether the characteristics differed according to primary malignancy.

Methods. We retrospectively analyzed data on 20 patients diagnosed with TBM between January 2000 and January 2017. Demographics, the period from diagnosis of primary malignancy to TBM diagnosis, the period from TBM diagnosis to death, the type and staging of primary malignancy, otologic manifestations, and TBM sites were assessed. After the primary malignancies were divided into solid cancers and hematologic malignancies, each parameter was compared between the two groups.

Results. The most common primary malignancy with TBM was lung cancer (45\%). The most common otologic symptoms and signs were facial palsy $(30.5 \%)$ and hearing loss $(30.5 \%)$. The temporal squama $(23 \%)$ and the facial nerve $(20 \%)$ were the most commonly involved. Most TBMs occurred late in the disease process after the primary malignancy first metastasized to other organs. Hematologic malignancies metastasized significantly more frequently to the external auditory canal and the middle ear/mastoid compared to solid cancers ( $P=0.001$ and $P=0.004$, respectively).

Conclusion. If otologic manifestations such as facial palsy and hearing loss are presented in patients at advanced stages of malignancy, TBM of primary malignancy should be suspected. In addition, hematologic malignancies tend to metastasize to the external auditory canal and the middle ear cleft more commonly than solid cancers do.

Keywords. Temporal Bone; Neoplasm Metastasis; Head and Neck Neoplasms; Leukemia

\section{INTRODUCTION}

Malignancy of the temporal bones is very rare [1]. Metastases of primary malignancies to the temporal bones are known to be less common than temporal bone cancer. To date, the true incidence of temporal bone metastasis (TBM) is not yet well known $[2,3]$. However, the incidence seems to be gradually increasing because of increased cancer survival rates and the development of diagnostic techniques.

Various types of malignancies can metastasize to the tempo-

- Received February 8, 2018

Revised April 13, 2018

Accepted May 4, 2018

- Corresponding author: Jin Woong Choi

Department of Otorhinolaryngology-Head and Neck Surgery, Chungnam

National University College of Medicine, 282 Munhwa-ro, Jung-gu,

Daejeon 35015, Korea

Tel: +82-42-280-8375, Fax: +82-42-253-4059, E-mail: choijw@cnu.ac.kr ral bone. It has been reported that not only solid cancers, such as lung cancer and breast cancer [4], but hematologic malignancies, such as leukemia, also spread to the temporal bone [5]. TBM occurs predominantly through hematologic spread, but may be caused by direct extension, meningeal carcinomatosis, or leukemic infiltration [6]. To date, most studies on TBM have been case or postmortem studies on temporal bones [7-9]. Therefore, there are some difficulties in fully understanding the clinical features of TBM. It is also unclear whether there are any clinical differences between TBM from solid cancers and hematologic malignancies.

The purposes of this study were to analyze the clinical features of TBM and to determine whether there were any differences in the characteristics according to type of primary malignancy, which was divided into solid cancers and hematologic malignancies.

Copyright (C) 2019 by Korean Society of Otorhinolaryngology-Head and Neck Surgery.

This is an open-access article distributed under the terms of the Creative Commons Attribution Non-Commercial License (http://creativecommons.org/licenses/by-nc/4.0)

which permits unrestricted non-commercial use, distribution, and reproduction in any medium, provided the original work is properly cited. 


\section{MATERIALS AND METHODS}

We retrospectively analyzed data on 20 patients diagnosed with TBM between January 2000 and January 2017 at the Department of Otolaryngology, Chungnam National University Hospital. Sixteen of the 20 patients were diagnosed based on histopathologic examination and the remaining four patients were diagnosed based on clinical symptoms, signs, and radiologic examination. Radiologically, TBM was defined when all three of the following imaging findings were satisfied: low-signal intensity in the T1-weighted image, low- or iso-signal intensity in the T2-weighted image, and contrast enhancement in the gadolinium-enhanced T1-weighted image. The study was approved by Institutional Review Board of Chungnam National University Hospital (IRB No. CNUH 2015-10-046) and informed consent was waived.

In each patient, the period from diagnosis of primary malignancy to diagnosis of TBM, the period from diagnosis of TBM to death, the type and staging of primary malignancy, symptoms and signs related to the ear, and sites of TBM were assessed. The metastatic sites to the temporal bone were classified as the temporal squama, petrous apex, internal auditory canal, external auditory canal, facial nerve, and middle ear cleft including the middle ear cavity and mastoid air cell. Cancer staging was classified according to the National Comprehensive Cancer Network guidelines for solid cancers and acute myeloid leukemia (AML). Cases of acute lymphocytic leukemia (ALL) were classified according to the presence of the Philadelphia chromosome. In addition, we compared these parameters after dividing the primary malignancies into solid cancers and hematologic malignancies to determine the differences between the two groups.

Statistical analysis was performed using the Mann-Whitney test for assessing the significance of differences in parametric data and Fisher exact test for analyzing nonparametric data. Differences were considered significant for probability values of $P<0.05$. All statistical analyses were performed using IBM SPSS ver. 22.0 (IBM Corp.,Armonk, NY, USA).

\section{H I G GHLLII G H T TS}

- The most common primary malignancy with temporal bone metastasis (TBM) was lung cancer and the most common otologic manifestations were facial palsy and hearing loss which were similar to those of chronic otitis media.

- Most TBMs occurred late in the disease process after the primary malignancy first metastasized to other organs.

- If otologic manifestations such as facial palsy and hearing loss are presented in patients at advanced stages of malignancy, TBM of primary malignancy should be suspected.

- Hematologic malignancies tend to metastasize to the external auditory canal and the middle ear cleft more commonly than solid cancers do.

\section{RESULTS}

\section{Demographics and primary malignancy}

The median age of the 20 patients with TBM was 60 years (range, 25 to 78 years), with 13 males and seven females. Among them, $16(80 \%)$ had solid cancers and the remaining four $(20 \%)$ had hematologic malignancies. Of the 16 solid cancers, nine $(56.3 \%)$ were lung cancers, which was the most common primary cancer with TBM. There were two $(12.5 \%)$ each of breast, gastric, and renal cancer, and there was one bladder cancer. Of the four hematologic malignancies, three were AML and one was ALL. Of the 14 solid cancers for which the clinical staging could be established, all except for two patients were at stage IV that already exhibited distant metastases to other systems. All cases of leukemia were classified as poor prognosis cases that had either the Philadelphia chromosome or higher risk factors (Table 1).

\section{Otologic manifestations and sites of metastases}

Of the total 20 patients, 17 showed otologic symptoms and signs, while three were asymptomatic. In patients with otologic manifestations, facial palsy and hearing loss were the most common symptoms and signs, which accounted for $30.5 \%$ each. Otorrhea (13\%) was the next most common symptom (Fig. 1A). In terms of sites of metastases, the temporal squama and facial nerve were metastatic sites in eight $(23 \%)$ and seven $(20 \%)$ patients, respectively, followed by the internal and external auditory canals, petrous apex, and middle ear cleft (Fig. 1B).

\section{Periods from diagnosis of primary malignancy to diagnosis of TBM and from diagnosis of TBM to death}

The median duration from diagnosis of primary carcinoma to
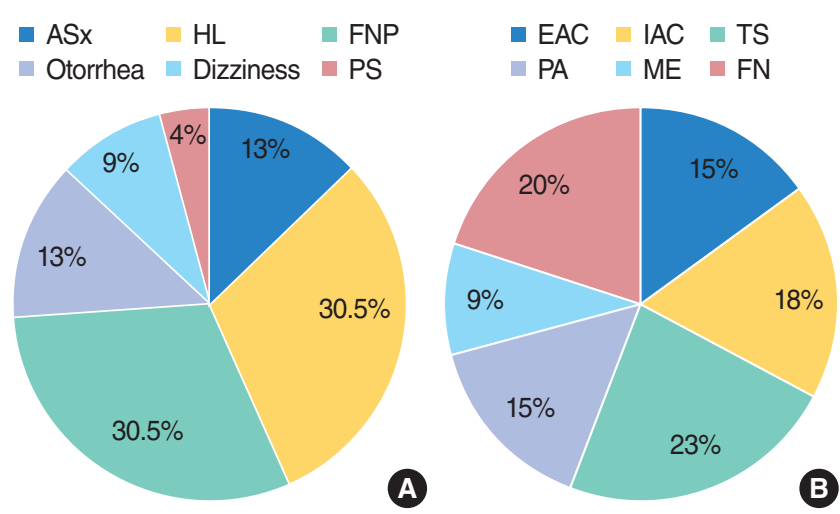

Fig. 1. Otologic manifestations and sites of temporal bone metastases (TBMs). (A) Hearing loss ( $\mathrm{HL}$ ) and facial nerve palsy (FNP) were common symptoms and signs of TBM. (B) The temporal squama (TS) and the facial nerve (FN) were common sites of TBM. ASx, asymptomatic; PS, periauricular swelling; EAC, external auditory canal; IAC, internal auditory canal; PA, petrous apex; ME, middle ear cleft. 
Table 1. Summary of patient's demographics, staging, otologic symptoms, and site of TBM

\begin{tabular}{|c|c|c|c|c|c|c|}
\hline No. & Sex & Age (yr) & Primary cancer & $\begin{array}{l}\text { Primary cancer staging } \\
\text { at diagnosis of TBM }\end{array}$ & Otologic symptom & TBM site \\
\hline 1 & Female & 77 & Lung cancer & T4N3M0, stage IV & ASx & PA \\
\hline 2 & Male & 53 & Renal cell carcinoma & T2aN0M1, stage IV & Periauricular swelling & TS \\
\hline 3 & Male & 67 & Lung cancer & T4N3M1, stage IV & ASx & TS \\
\hline 4 & Female & 60 & Breast cancer & T2N1M0, stage Ilb & $\mathrm{HL}, \mathrm{FNP}$ & $\mathrm{FN}$ \\
\hline 5 & Male & 60 & Gastric cancer & TxN3aM1, stage IV & Dizziness & $\mathrm{IAC}$ \\
\hline 6 & Female & 63 & Lung cancer & T1bNOM0, stage IA & $\mathrm{HL}$ & $\mathrm{IAC}$ \\
\hline 7 & Male & 55 & Lung cancer & NA & FNP & TS, FN \\
\hline 8 & Female & 64 & Lung cancer & T3N3M1a, stage IV & $\mathrm{HL}$ & IAC \\
\hline 9 & Female & 48 & Lung cancer & T3N1M1, stage IV & ASx & TS \\
\hline 10 & Male & 73 & Lung cancer & T2aNOM1, stage IV & FNP & $\mathrm{PA}, \mathrm{IAC}, \mathrm{FN}$ \\
\hline 11 & Female & 41 & Breast cancer & NA & FNP & PA, FN, TS \\
\hline 12 & Male & 51 & Bladder cancer & T2N2M1, stage IV & Otorrhea & EAC \\
\hline 13 & Male & 72 & Lung cancer & T3N3M1b, stage IV & $\mathrm{HL}$ & TS \\
\hline 14 & Male & 38 & ALL & Philadelphia chromosome positive & Otorrhea, FNP & EAC, FN, PA, ME \\
\hline 15 & Male & 35 & Gastric cancer & T4N3M1, stage IV & FNP & $\mathrm{PA}, \mathrm{IAC}, \mathrm{FN}$ \\
\hline 16 & Male & 77 & AML & Intermediate risk group & $\mathrm{HL}$ & $\mathrm{EAC}, \mathrm{ME}$ \\
\hline 17 & Male & 74 & AML & Intermediate risk group & $\mathrm{HL}$ & EAC \\
\hline 18 & Female & 57 & Lung cancer & T2aN3M1b, stage IV & Dizziness & IAC, TS \\
\hline 19 & Male & 25 & AML & Poor risk group & Otorrhea, FNP & EAC, FN, ME \\
\hline 20 & Male & 78 & Renal cell carcinoma & T4NOMO, stage IV & $\mathrm{HL}$ & TS \\
\hline
\end{tabular}

TBM, temporal bone metastasis; ASx, asymptomatic; PA, petrous apex; TS, temporal squama; HL, hearing loss; FNP, facial nerve palsy; FN, facial nerve; IAC, internal auditory canal; NA, not applicable; EAC, external auditory canal; ALL, acute lymphocytic leukemia; ME, middle ear cleft; AML, acute myeloid leukemia.

TBM was 22.5 months (range, 0 to 127.0 months). Of the 20 patients, 15 (75\%) died from the malignancies and four patients $(20 \%)$ were alive with disease. There was one case of follow-up loss. One patient harbored primary malignancy and TBM simultaneously. The median time to death from the diagnosis of TBM was 3.0 months (range, 0.8 to 30.0 months), which was significantly shorter than the period from diagnosis of primary malignancy to TBM $(P=0.011)$ (Fig. 2, Table 2$)$. There was no association between the period from diagnosis of primary malignancy to diagnosis of TBM and multiplicity of TBM sites $(P=0.083)$

\section{Solid cancers vs. hematologic malignancies}

There were no demographic differences between TBM from solid cancers and hematologic malignancies. The periods from primary cancer to TBM and from TBM to death were similar in both groups. Those with hematologic malignancies exhibited metastases more commonly to the external auditory canal and middle ear cleft compared to those with solid cancers, which were statistically significant $(P=0.001$ and $P=0.004$, respectively). There were no significant differences between the two groups in metastases to other sites including the internal auditory canal, temporal squama, petrous apex, and facial nerve. There were also no differences in the number of metastatic sites between the two groups (Table 2).

\section{DISCUSSION}

Various types of cancer are known to cause metastases to the temporal bones. In this study, there were patients with lung, kidney, breast, gastric, and bladder cancer and leukemia, such as AML and ALL, as primary malignancies with TBM. Among them, lung cancer was the most common primary cancer. This result is similar with a previous study that demonstrated that about $20 \%$ of TBM, including those in the internal auditory canal, resulted from lung cancer [10]. However, other studies have shown that kidney cancer or breast cancer was the most common primary cancer with TBM $[11,12]$. Lung, kidney, and breast cancers must share common properties such that they have a predilection for disseminating to the bone [13]. The differences in incidence may be due to the country or region in which the respective study was conducted. Lung cancer is a major primary cancer in countries with a large number of smokers, while breast cancer is a common primary malignancy in developed countries than in developing countries.

We noted that 16 out of the 18 patients in whom cancer staging could be determined were either at an advanced stage or at high risk. In addition, 12 out of 16 patients with solid cancers had other systemic metastases before TBM. Therefore, TBM seems to occur late in the disease process after the primary cancers first metastasize to other organs.

The most common clinical manifestations associated with 


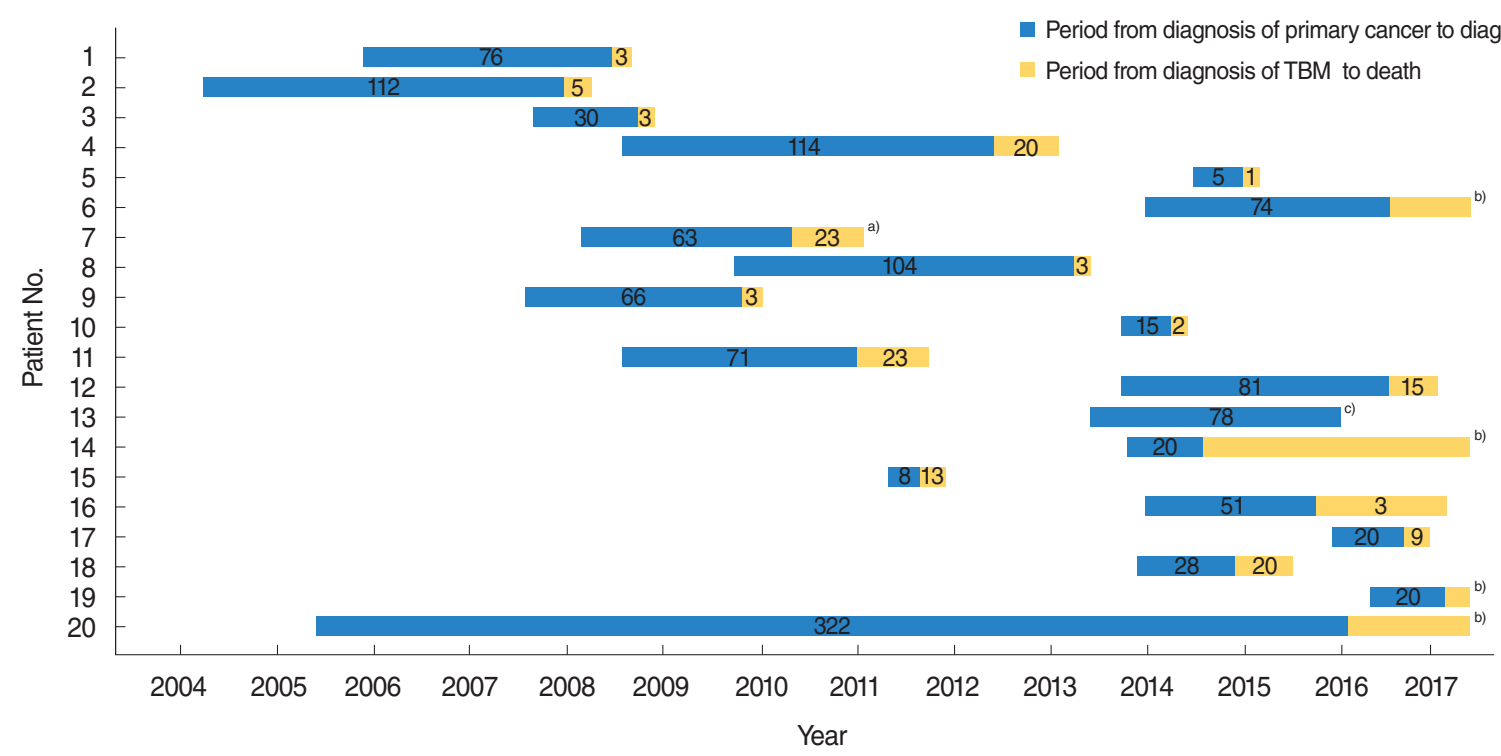

Fig. 2. Gantt chart showing periods (months) from diagnosis of primary malignancy to temporal bone metastasis (TBM) and from TBM to death. The time from the diagnosis of TBM to death was significantly shorter than the period from diagnosis of primary malignancy to TBM $(P=0.011) .{ }^{a}$ Lost to follow-up. ${ }^{\text {b) }}$ Alive with TBM. ${ }^{c}$ Diagnosis of TBM and primary malignancy at the same time.

Table 2. Comparison of clinical features of temporal bone metastases between solid cancers and hematologic malignancies

\begin{tabular}{|c|c|c|c|c|}
\hline Valuable & $\begin{array}{c}\text { Total } \\
(n=20)\end{array}$ & $\begin{array}{l}\text { Solid cancer } \\
\qquad(n=16)\end{array}$ & $\begin{array}{c}\text { Hematologic } \\
\text { malignancy }(n=4)\end{array}$ & $P$-value \\
\hline Age (yr) & $\begin{array}{c}60 \\
(25-78)\end{array}$ & $\begin{array}{c}60 \\
(35-78)\end{array}$ & $\begin{array}{c}56 \\
(25-77)\end{array}$ & 0.820 \\
\hline Sex (male:female) & $13: 7$ & $9: 7$ & $4: 0$ & 0.249 \\
\hline $\begin{array}{l}\text { Period from primary } \\
\text { cancer to TBM } \\
\text { (mo) }\end{array}$ & $\begin{array}{c}22.5 \\
(0-127.0)\end{array}$ & $\begin{array}{c}27.0 \\
(0-127.0)\end{array}$ & $\begin{array}{c}8.0 \\
(8.0-20.0)\end{array}$ & 0.211 \\
\hline $\begin{array}{l}\text { Period from TBM to } \\
\text { death }(\mathrm{mo})\end{array}$ & $\begin{array}{c}3.0 \\
(0.8-30.0)\end{array}$ & $\begin{array}{c}2 \\
(0.8-30.0)\end{array}$ & $\begin{array}{c}9.5 \\
(3.0-16.0)\end{array}$ & 0.381 \\
\hline Metastases sites & & & & 0.255 \\
\hline Single & 12 & 11 & 1 & \\
\hline Multiple & 8 & 5 & 3 & \\
\hline EAC involvement & & & & 0.001 \\
\hline Yes & 5 & 1 & 4 & \\
\hline No & 15 & 15 & 0 & \\
\hline IAC involvement & & & & 0.267 \\
\hline Yes & 6 & 6 & 0 & \\
\hline No & 14 & 10 & 4 & \\
\hline TS involvement & & & & 0.117 \\
\hline Yes & 8 & 8 & 0 & \\
\hline No & 12 & 8 & 4 & \\
\hline PA involvement & & & & 1.000 \\
\hline Yes & 5 & 4 & 1 & \\
\hline No & 15 & 12 & 3 & \\
\hline ME involvement & & & & 0.004 \\
\hline Yes & 3 & 0 & 3 & \\
\hline No & 17 & 16 & 1 & \\
\hline FN involvement & & & & 0.587 \\
\hline Yes & 7 & 5 & 2 & \\
\hline No & 13 & 11 & 2 & \\
\hline
\end{tabular}

Values are presented as median (range) or number.

TBM, temporal bone metastases; EAC, external auditory canal; IAC, internal auditory canal; TS, temporal squama; PA, petrous apex; ME, middle ear cleft; FN, facial nerve.
TBM in the present study were facial palsy and hearing loss. These results are similar to those obtained in previous studies. Schuknecht et al. [14] reported that $14.9 \%$ of patients with TBM showed facial palsy, which was slight lower than our incidence. Gloria-Cruz et al. [11] reported that hearing loss was found in $40.4 \%$ of their subjects, which was the most common symptom in their study. Facial palsy and hearing loss are also common clinical manifestations of chronic otitis media. Therefore, if symptoms similar to chronic otitis media occur in patients with cancer, TBM of primary malignancy should be suspected.

In addition, none of our patients presented with the triad of symptoms (otalgia, periauricular swelling, and facial nerve paresis) as suggested by Maddox [12], possibly because the symptom triad may have occurred when TBM had progressed considerably. Interestingly, only four patients had headache at the time of TBM diagnosis. Otologic symptoms such as facial palsy and hearing loss seem to be more important clues in TBM diagnosis than skull base symptoms such as headache.

We found that the temporal squama $(23 \%)$ and the facial nerve $(20 \%)$ were frequently involved in TBM. The internal auditory canal $(18 \%)$ was also a common metastatic site. These metastatic sites coincided well with the clinical symptoms and signs. Previous studies have reported that the petrous apex was the most common metastatic site because not only is the petrous apex abundant in blood supply, but TBM also occurs mainly through hematogenous spread $[4,11]$. Interestingly, no metastasis to the inner ear was found in the current study. GloriaCruz et al. [11] also reported that none of their 76 autopsy specimens had an involvement of the inner ear. This might be due to the poor vascularity of the inner ear including the otic 
capsule and membranous labyrinth compared to other parts of the temporal bone [2].

Although it has been previously reported that TBM to the internal auditory canal occurs many decades after primary cancer diagnosis [15], we observed that the time from diagnosis of primary malignancy to diagnosis of TBM was an average of 22.5 months. The median time to death after diagnosis of TBM was 3.0 months, which was significantly shorter than the period from primary cancer diagnosis to diagnosis of TBM.

All patients received either chemotherapy or radiotherapy after TBM was diagnosed; however, all but four died. The majority of reports have also described poor prognosis with short survival times, similar to those shown in the present study [8]. The prognosis could be attributed to poor general conditions and surgical difficulties in accessing sites of TBM.

We found that hematologic malignancies exhibit significantly more metastases to the external auditory canal and middle ear cleft than solid cancers do. Although the exact mechanism is unknown, this may be due to differences in metastatic patterns. Hematologic malignancies can spread to other organs by leukemic infiltration with submucous hemorrhage as well as hematogenous spread, whereas solid cancers mainly disseminate to distant sites via hematogenous pathways $[6,11]$. A histopathologic study showed that submucous hemorrhage and leukemic infiltration were major findings in temporal bones from patients with leukemia [16]. In addition, infiltration and submucous hemorrhage in the external auditory canal are likely to be easier observed than those in other parts of the temporal bones because, anatomically, the external auditory canal is externally exposed.

This study has several limitations. First, the number of subjects was small. In particular, the number of patients with hematologic malignancies was small. Therefore, a further large-scale study is required to verify our findings. Second, the design of the study was retrospective. In addition, the sites of metastases were determined by imaging studies in areas where the biopsy could not be performed. Autopsy may have revealed different results. However, the majority of patients with TBM also have other systemic metastases, so they are in poor general condition and are unable to undergo biopsy or surgery. Some parts of the temporal bones, such as the petrous apex and the internal auditory canal, are also not easy to access. Therefore, the diagnosis of TBM in clinical settings is dependent on imaging tests. In the present study, four out of a total of 20 patients $(20 \%)$ were diagnosed with TBM based on imaging studies, while the other 16 patients were confirmed to have metastases based on histologic examination.

In conclusion, the most common primary cancer that results in TBM is lung cancer. The metastases frequently present with facial palsy and hearing loss, which are similar with those of chronic otitis media. In addition, TBM is likely to occur when the primary cancer is advanced and has metastasized to other systems. Therefore, if otologic symptoms and signs, such as facial palsy or hearing loss, occur in patients with advanced primary cancer, we should have a high index of suspicion of TBM. Furthermore, hematologic malignancies tend to frequently disseminates to the external auditory canal and middle ear cleft compared to solid cancers.

\section{CONFLICT OF INTEREST}

No potential conflict of interest relevant to this article was reported.

\section{ACKNOWLEDGMENTS}

This work was supported by Chungnam National University Hospital Research Fund, Daejeon, Korea in 2013.

\section{REFERENCES}

1. Streitmann MJ, Sismanis A. Metastatic carcinoma of the temporal bone. Am J Otol. 1996 Sep;17(5):780-3.

2. Jahn AF, Farkashidy J, Berman JM. Metastatic tumors in the temporal bone: a pathophysiologic study. J Otolaryngol. 1979 Feb;8(1):8595.

3. Nelson EG, Hinojosa R. Histopathology of metastatic temporal bone tumors. Arch Otolaryngol Head Neck Surg. 1991 Feb;117(2):18993.

4. Belal A Jr. Metastatic tumours of the temporal bone: a histopathological report. J Laryngol Otol. 1985 Sep;99(9):839-46.

5. Aljafar HM, Alsuhibani SS, Alahmari MS, Alzahrani MA. Temporal bone metastasis as a sign of relapsing chronic lymphocytic leukemia. Saudi Med J. 2015 Oct;36(10):1233-5.

6. Berlinger NT, Koutroupas S, Adams G, Maisel R. Patterns of involvement of the temporal bone in metastatic and systemic malignancy. Laryngoscope. 1980 Apr;90(4):619-27.

7. De Vos C, Gerard JM, Decat M, Gersdorff M. Metastatic renal cell carcinoma to the temporal bone: case report. B-ENT. 2005;1(1):43-6.

8. Pontious MB, Kim SY, Backous DD. Metastasis to the petrous apex: a report of an uncommon case. Otolaryngol Head Neck Surg. 2003 Dec;129(6):751-3.

9. Weiss MD, Kattah JC, Jones R, Manz HJ. Isolated facial nerve palsy from metastasis to the temporal bone: report of two cases and a review of the literature. Am J Clin Oncol. 1997 Feb;20(1):19-23.

10. Chang MT, Michaelides EM. High rate of bilaterality in internal auditory canal metastases. Am J Otolaryngol. 2015 Nov-Dec;36(6): 798-804.

11. Gloria-Cruz TI, Schachern PA, Paparella MM,Adams GL, Fulton SE. Metastases to temporal bones from primary nonsystemic malignant neoplasms. Arch Otolaryngol Head Neck Surg. 2000 Feb;126(2): 209-14.

12. Maddox HE 3rd. Metastatic tumors of the temporal bone. Ann Otol Rhinol Laryngol. 1967 Mar;76(1):149-65.

13. Sahin AA, Ro JY, Ordonez NG, Luna MA, Weber RS, Ayala AG. Temporal bone involvement by prostatic adenocarcinoma: report of two cases and review of the literature. Head Neck. 1991 Jul-Aug;13(4): 349-54. 
14. Schuknecht HF, Allam AF, Murakami Y. Pathology of secondary malignant tumors of the temporal bone. Ann Otol Rhinol Laryngol. 1968 Feb;77(1):5-22.

15. Della Puppa A, Rossetto M, Berti F, Zustovich F, Manara R, Gardiman MP, et al. Internal auditory canal metastasis. J Neurosurg Sci.
2010 Dec;54(4):159-62.

16. Terao K, Cureoglu S, Schachern PA, Paparella MM, Morita N, Nomiya S, et al. Pathologic correlations of otologic symptoms in acute lymphocytic leukemia. Am J Otolaryngol. 2011 Jan-Feb;32(1):13-8. 